\title{
Photonic and Plasmonic Guided Modes in Graphene-Silicon Photonic Crystals
}

Gu, Tingyi; Andryieuski, Andrei; Hao, Yufeng; Li, Yilei; Hone, James; Wong, Chee Wei; Lavrinenko, Andrei; Low, Tony; Heinz, Tony F.

Published in:

A C S Photonics

Link to article, DOI:

10.1021/acsphotonics.5b00209

Publication date:

2015

Document Version

Publisher's PDF, also known as Version of record

Link back to DTU Orbit

Citation (APA):

Gu, T., Andryieuski, A., Hao, Y., Li, Y., Hone, J., Wong, C. W., Lavrinenko, A., Low, T., \& Heinz, T. F. (2015). Photonic and Plasmonic Guided Modes in Graphene-Silicon Photonic Crystals. A C S Photonics, 2(11), 1552 1558. https://doi.org/10.1021/acsphotonics.5b00209

\section{General rights}

Copyright and moral rights for the publications made accessible in the public portal are retained by the authors and/or other copyright owners and it is a condition of accessing publications that users recognise and abide by the legal requirements associated with these rights.

- Users may download and print one copy of any publication from the public portal for the purpose of private study or research.

- You may not further distribute the material or use it for any profit-making activity or commercial gain

- You may freely distribute the URL identifying the publication in the public portal 


\title{
Photonic and Plasmonic Guided Modes in Graphene-Silicon Photonic Crystals
}

\author{
Tingyi Gu, ${ }^{\dagger, \dagger}$ Andrei Andryieuski, ${ }^{\dagger} \S$ Yufeng Hao, ${ }^{\|}$Yilei Li, ${ }^{\perp}$ James Hone, ${ }^{\|}$Chee Wei Wong, ${ }^{\|, \#}$ \\ Andrei Lavrinenko, ${ }^{\S}$ Tony Low, $*,+, \square$ and Tony F. Heinz ${ }^{\ddagger, \perp, O}$
}

${ }^{\ddagger}$ Department of Electrical Engineering, "Department of Mechanical Engineering, and ${ }^{\perp}$ Department of Physics, Columbia University, New York, New York 10027, United States

${ }^{\S}$ DTU Fotonik, DTU, Oersteds pl. 343, Kongens Lyngby, Denmark 2800

${ }^{\#}$ Mesoscopic Optics and Quantum Electronics Laboratory, University of California, Los Angeles, California 90095, United States

ODepartments of Applied Physics and Photon Science, Stanford University, Stanford, California 94305, United States

\section{Supporting Information}

ABSTRACT: We report the results of systematic studies of plasmonic and photonic guided modes in large-area singlelayer graphene integrated into a nanostructured silicon substrate. The interaction of light with graphene and substrate photonic crystals can be classified in distinct regimes depending on the relation of the photonic crystal lattice constant and the relevant modal wavelengths, that is, plasmonic, photonic, and free-space. By optimizing the design of the substrate, these resonant modes can increase the absorption of graphene in the infrared, facilitating enhanced performance of modulators, filters, sensors, and photodetectors utilizing silicon photonic platforms.

KEYWORDS: graphene absorption, graphene plasmonic response, silicon photonics, infrared response, absorption enhancement
$\mathrm{T}$ he continuous miniaturization of components in silicon photonics provides a platform to enable applications in densely integrated communication and computing systems. ${ }^{1}$ The high refractive index and low absorption of silicon allow for low-loss waveguiding from the terahertz to the telecommunication spectral range in the near-infrared, but its band gap of $1.1 \mathrm{eV}$ renders silicon an inefficient material for photodetection in this long wavelength range. Hence, photonic systems based on alternative materials, such as III-V semiconductors $^{2}$ and graphene, ${ }^{3}$ are sought for those applications. ${ }^{4}$ In particular, graphene is a promising material since it supports mid-infrared plasmons ${ }^{5,6}$ with light confinement down to $1 / 100$ of the free-space wavelength $\lambda_{0}$, as well as tunability of electrical conductivity, thus, allowing the creation of active devices ${ }^{7}$ not possible with conventional metal plasmonics. Graphene plasmons have already been experimentally observed ${ }^{7-12}$ and explored for terahertz and infrared absorption, modulation, photodetection, and chemical sensing. ${ }^{13-18}$ Furthermore, graphene can be easily integrated with silicon photonics components for more efficient light management schemes. ${ }^{14,19}$

One of the challenges for the excitation and use of surface plasmon polaritons in graphene is the phase (wavevector) mismatch between these guided waves and the incident electromagnetic radiation. Graphene nanostructures, such as one-dimensional (1D) nanoribbon arrays ${ }^{11,12,20}$ and twodimensional (2D) rectangular resonator arrays, ${ }^{21} \operatorname{dot}^{7}$ and antidot lattices, ${ }^{22,23}$ and plasmonic crystals, ${ }^{24}$ provide a natural approach to coupling free-space radiation to graphene plasmons. However, these schemes require that the graphene layer be patterned, which introduces additional loss channels from atomic scale roughness of the edges. ${ }^{11,25}$ It is therefore attractive to couple to graphene plasmons by the alternate approach of patterning the substrate, leaving the graphene layer in its pristine state and preserving its excellent electronic properties.

The case of a $1 \mathrm{D}$ grating etched into a silicon substrate with a graphene overlayer has been studied theoretically for mid-IR plasmon excitation ${ }^{26}$ and subsequently investigated experimentally in a hexagonal 2D grating configuration. ${ }^{27}$ These configurations exploit the difference in the effective mode index between plasmons in a silicon-graphene-air structure (graphene on silicon) and in a suspended air-graphene-air structure (graphene over holes), in addition to using the associated contrast of the dynamic conductivity. ${ }^{28}$ At the same time, photonic modes within the silicon grating can also be excited. These photonic modes can be used to increase the absorbance in graphene, as was recently demonstrated. ${ }^{29-33}$ In this work, we explore the various regimes of light-matter interaction in the infrared spectral range for graphene integrated with a silicon photonic crystal membrane. We

Received: April 21, 2015

Published: October 5, 2015 
a

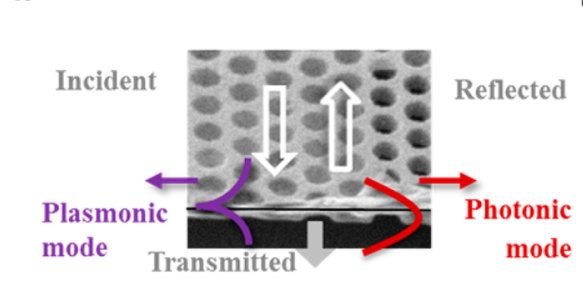

b

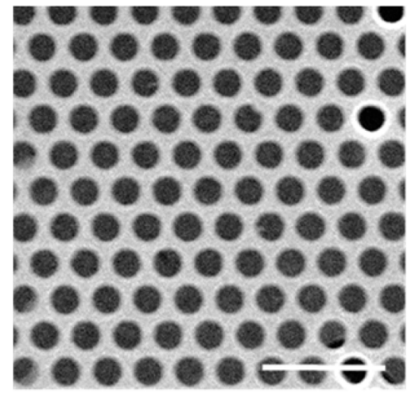

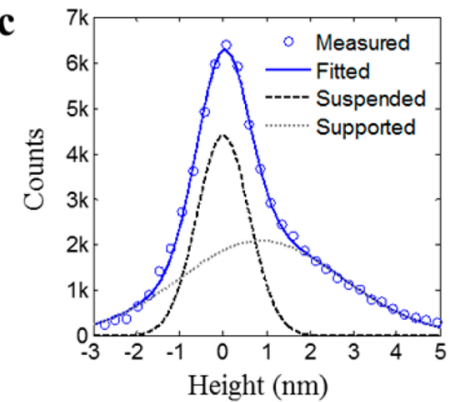

d
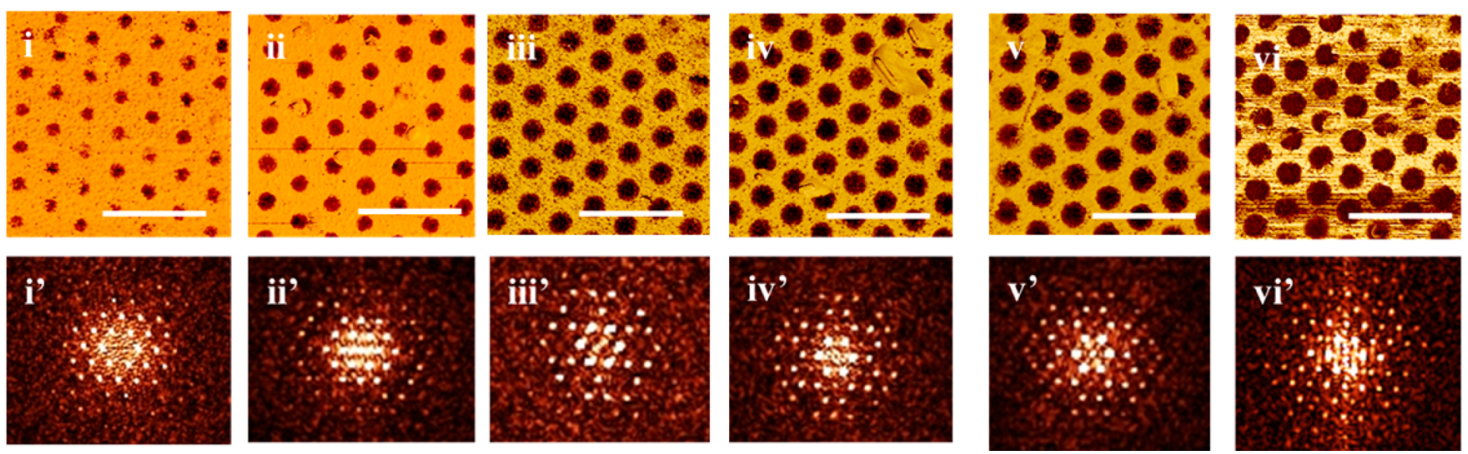

Figure 1. Suspended graphene superlattice. (a) SEM image combined with a sketch of the plasmonic and photonic modes excited by a normally incident plane wave. (b) SEM top view of the graphene-photonic crystal structure. (c) Histogram of the topology measured by AFM. The experimental peak is decomposed into a part corresponding to suspended graphene (over holes, fwhm of $1.72 \mathrm{~nm}$ ) and to supported graphene (on silicon, fwhm of $5.31 \mathrm{~nm}$ ), with a $0.57 \mathrm{~nm}$ height difference. (d) AFM image of the surface stiffness of graphene on a silicon photonic crystal membrane for different disk radii: $r=$ (i) 90, (ii) 100, (iii) 110, (iv) 120, (v) 130, and (vi) $140 \mathrm{~nm}$ and the corresponding 2D spatial Fourier transforms $\left(\mathrm{i}^{\prime}-\mathrm{vi}^{\prime}\right.$, respectively). The lattice constant is $a=415 \mathrm{~nm}$ and the scale bars is $1 \mu \mathrm{m}$ in length.

demonstrate experimentally the possibility of light coupling to the graphene plasmonic modes and to the photonic guided modes of the underlying substrate. We examine theoretically the physical mechanisms and factors controlling the crossover between these regimes.

\section{STRUCTURES INVESTIGATED AND THEIR CLASSIFICATION}

A scanning electron micrograph of a representative structure consisting of graphene on a photonic crystal membrane is presented in Figure 1a. The different regimes of light coupling to the structure can be categorized based on the feature size of the structure (particularly the lattice constant $a$ ), the effective wavelengths for the plasmonic $\left(\lambda_{\mathrm{pl}}\right)$ and photonic crystal $\left(\lambda_{\mathrm{pc}}\right)$ modes, and the incident (vacuum) wavelength of the light $\left(\lambda_{0}\right)$. We consider the typical regime for which $\lambda_{\mathrm{pl}}<\lambda_{\mathrm{pc}}<\lambda_{0}$ and assume incident light from the vacuum. We identify four regimes of interest:

1. $a<\lambda_{\mathrm{pl}}$ : Metamaterial Regime. In order to excite a guided wave efficiently at normal incidence, the distance between scatterers in a periodic array should be equal to an integral multiple of the effective wavelength of the guided mode. This condition clearly cannot be met in the of $a<\lambda_{\text {pl }}$ and we can consequently consider graphene as a plasmonic metamaterial (or metasurface), since no guided surface waves can be excited due to the pronounced phase mismatch. The incident wave can only be reflected, transmitted, and absorbed in the graphene layer. (Absorption in silicon substrate is low for the relevant spectral range.) The structured silicon substrate can be considered as a homogeneous layer with an anisotropic dielectric response. ${ }^{34}$

2. $\lambda_{\mathrm{pl}}<a<\lambda_{\mathrm{pc}}$ : Plasmonic Regime. In this regime, in addition to transmission and reflection of the incident radiation, coupling of the incident light to plasmonic modes becomes possible at certain frequencies. This coupling occurs when phase matching is achieved, i.e., for incident waves with propagation constant $\beta=q k_{0}=(2 \pi / a) m$, where $q$ is the effective mode index, $k_{0}=2 \pi / \lambda_{0}$ is the free-space wavevector of the light, and $m$ is an integer. The incident wave couples to graphene plasmons that are tightly confined by the graphene layer. Their energy is partially absorbed by the graphene and partially coupled back to free space.

3. $\lambda_{\text {pc }}<a<\lambda_{0}$ : Photonic Regime. In addition to the aforementioned channels, in this regime, the incident wave can couple to the photonic modes of the membrane. Radiation is then absorbed by graphene, which acts mostly as a lossy medium. The photonic modes depend not only on the grating lattice constant, but also on the thickness of the silicon layer. Since we assume light coupling from a normally incident plane wave, we are concerned with the photonic modes that are normally considered as leaky.

4. $\lambda_{0}<a$ : Diffraction Grating Regime. In this regime, the periodic structure becomes a diffraction grating and the incident power is distributed among various diffraction orders, in addition to excitation of the photonic and plasmonic modes described above.

We note that for sufficiently large lattice constant $a$, all these types of coupling are possible simultaneously, though they are usually important in different frequency ranges. The metamaterial regime for absorbers is well-known, ${ }^{35}$ while the diffraction grating regime is not particularly suitable for absorbers, since the incident power is predominantly scattered back into free space. In this work, we consequently explore both experimentally and theoretically the most interesting plasmonic and photonic regimes (see illustration in Figure 1a). 
Hexagonal 2D silicon photonic crystal membranes were fabricated on a $250 \mathrm{~nm}$ thick silicon-on-insulator layer by deep ultraviolet photolithography $(248 \mathrm{~nm})$ and etched to reduce disorder scattering. ${ }^{36}$ The silicon layer is intrinsic, with an estimated doping level of $10^{15} \mathrm{~cm}^{-3}$. The lattice constant of the photonic crystal was chosen as $415 \mathrm{~nm}$, with hole radii varying from 80 to $140 \mathrm{~nm}$ in $10 \mathrm{~nm}$ steps, corresponding, respectively, to silicon filling fractions of $0.87-0.59$. The sacrificial release of the supporting silicon oxide buffer layer was performed by wet etching, resulting in a $0.5 \mu \mathrm{m}$ air gap between photonic crystal membrane and the underlying silica substrate (Figure S1).

Large-area graphene monolayers were grown on copper film by a chemical vapor deposition (CVD) process. ${ }^{37}$ The thickness and crystallinity of the graphene layers were characterized by means of Raman spectroscopy (data not shown). The graphene layers were then transferred onto patterned photonic crystal substrates by a PMMA-assisted method (Figure 1a,b). ${ }^{38}$ The resulting morphology of the graphene layers was examined by atomic force microscopy (AFM). These measurements yielded an apparent height difference of $0.89 \mathrm{~nm}$ between the regions of graphene supported by silicon and those suspended over holes (Figure 1c). The fwhm of the AFM peak provides an indication of the surface roughness of the graphene samples. Based on these measurements, the roughness of suspended and supported graphene layers was found to be 1.72 and $5.31 \mathrm{~nm}$, respectively, indicating that the transferred graphene was of reasonably good quality. The contrast in the surface stiffness of the suspended and supported regions of the graphene sample was used to map the overall structure (Figure $1 \mathrm{~d}$ ). The formation of graphenesilicon antidot arrays with a fixed lattice constant $a$ and increasing hole radii $r$ was identified by the surface stiffness mapping technique [Figure $1 \mathrm{~d}(\mathrm{i}-\mathrm{vi})]$. The hexagonal symmetry was present for all samples, with limited disorder from polymer residues, as shown in Figure $1 \mathrm{~d}(\mathrm{i}-\mathrm{vi})$ and in the corresponding two-dimensional Fourier transforms in Figure 1d $\left(\mathrm{i}^{\prime}-\mathrm{vi}^{\prime}\right)$.

In order to isolate the contribution of the graphene layer to modification of the optical response of the patterned substrate, we selectively etched away half of the graphene layer using an oxygen plasma (see Supporting Information, Figure S5a). We determined the precise position of the graphene on the substrate by Raman spectroscopy (Figure S5b), making use of the fact that a free-standing graphene monolayer exhibits a stronger Raman signal than that of a supported graphene monolayer. $^{39}$

For broadband optical characterization of the structure in the infrared, we probed the response of the sample using a commercial Fourier transform infrared spectrometer (Vertex, Bruker Optics), collecting the IR spectra in a reflection geometry. To this end broadband light from a thermal source was directed onto the sample at normal incidence through an aperture of $100 \mu \mathrm{m} \times 20 \mu \mathrm{m}$ size. We then measured the reflectance $R$ from the graphene-covered structure and the reflectance $R_{0}$ from an uncovered reference region of the photonic crystal. We characterize the influence of the graphene by its extinction ratio $E R$ inferred from the reflectivity measurements: $E R=1-R / R_{0}$.

The reflectance from the graphene-covered and uncovered reference structures (Figure S1) were simulated using the CST Microwave Studio package ${ }^{40}$ with the time-domain solver under the assumption of periodic boundary conditions. The relevant optical properties of silicon and silica (see Supporting
Information, Figure S2a,b) were taken from the refs 41 and 42, respectively. The response of the graphene monolayer was modeled as a sheet of thickness of $\Delta=1 \mathrm{~nm}$, with an effective dielectric permittivity of $\varepsilon_{\mathrm{G}}(\omega)=1+i \sigma_{\mathrm{S}}(\omega) / \varepsilon_{0} \omega \Delta$, where $\omega$ is the angular frequency and $\sigma_{\mathrm{S}}(\omega)$ is the sheet conductivity of graphene. For the latter, we made use of the expression from ref 43 (see Supporting Information for further details), which includes both the intra- and interband contributions to the conductivity and also accounts for the effects of finite temperature $T$ :

$$
\begin{aligned}
& \sigma_{\mathrm{S}}(\omega)=\frac{2 e^{2} k_{\mathrm{B}} T}{\pi \hbar^{2}} \ln \left[2 \cosh \left(\frac{E_{\mathrm{F}}}{2 k_{\mathrm{B}} T}\right)\right] \times \frac{i}{\omega+i \gamma} \\
& +\frac{e^{2}}{4 \hbar}\left[H\left(\frac{\omega}{2}\right)+\frac{4 i \omega}{\pi} \int_{\infty}^{0} \mathrm{~d} \frac{H(x)-H\left(\frac{\omega}{2}\right)}{\omega^{2}-4 x^{2}}\right] \\
& \text { where } H(x)=\frac{\sinh \left(\hbar x / k_{\mathrm{B}} T\right)}{\cosh \left(E_{\mathrm{F}} / k_{\mathrm{B}} T\right)+\cosh \left(\hbar x / k_{\mathrm{B}} T\right)} \\
& =\frac{1}{2}\left[\tanh \left(\frac{\hbar x+E_{\mathrm{F}}}{2 k_{\mathrm{B}} T}\right)+\tanh \left(\frac{\hbar x-E_{\mathrm{F}}}{2 k_{\mathrm{B}} T}\right)\right]
\end{aligned}
$$

In this description, there are two parameters describing the state of the graphene: the Fermi energy (or, rigorously, the chemical potential) $E_{\mathrm{F}}$ and the carrier scattering rate $\gamma$. In our simulations, we chose $E_{\mathrm{F}}=0.3 \mathrm{eV}$, a value comparable to the range of $0.25-0.35 \mathrm{eV}$ measured by terahertz spectroscopy for unintentionally doped and transferred graphene layers. ${ }^{44,45}$ For the scattering rate, we used $\gamma=2.0 \times 10^{13} \mathrm{~s}^{-1}$, close to experimentally measured value of $\gamma=2.3 \times 10^{13} \mathrm{~s}^{-1}$ for CVD graphene $^{46}$ (see Supporting Information, Figure S2c).

\section{PLASMONIC REGIME}

In the mid-IR spectral region, we expect light-matter interactions to be dominated by the plasmonic modes of graphene. For our structures, the lattice constant $a$ is much smaller than the wavelength of the light in this spectral range, so the membrane can be considered to be an effective homogeneous dielectric layer for the optical (but not plasmonic) waves (Supporting Information, Figure S2d, for the effective refractive index). For a free-space wavelength of $\lambda_{0}$ $=10 \mu \mathrm{m}\left(1000 \mathrm{~cm}^{-1}\right)$, the effective wavelengths for the plasmons in graphene are 352 and $54 \mathrm{~nm}$ for the supported and suspended regions, respectively, with corresponding propagation lengths of 264 and $41 \mathrm{~nm}$ (see Supporting Information, Figure S3).

Strong plasmonic coupling can occur if the size of a resonator in the structure is nearly equal to an integral number of effective wavelengths of the guided wave. In the case of a hole radius of $r$ $=100 \mathrm{~nm}$, the size of the silicon region between the edges of nearest neighboring holes is $215 \mathrm{~nm}$. Based on these simple considerations, we expect to be able to excite the second-order plasmonic mode (with an effective wavelength about $112 \mathrm{~nm}$ around $700 \mathrm{~cm}^{-1}$ ) at the silicon-graphene-air interface. (Excitation of the first-order mode would lie below $500 \mathrm{~cm}^{-1}$ and is consequently outside of our experimental spectral range.) Plasmons can also be excited at the air-graphene-air interface at higher frequencies, with the first-order resonance occurring at a frequency around $1200 \mathrm{~cm}^{-1}$. Due to the short propagation 

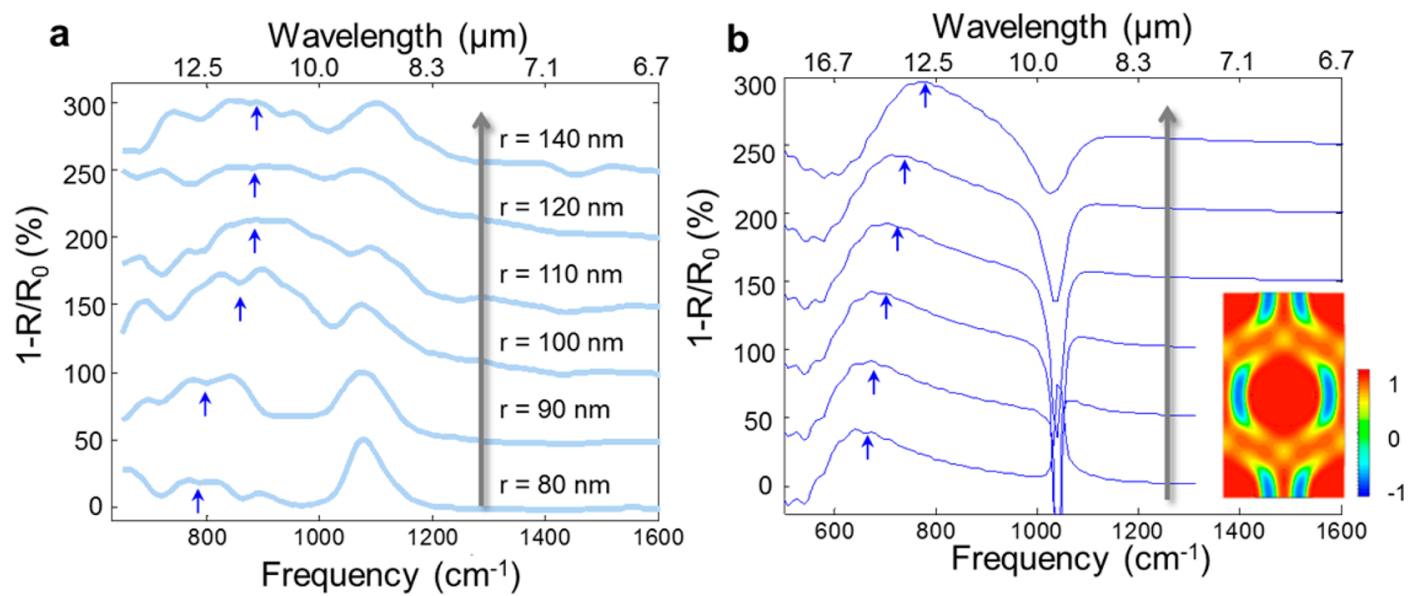

Figure 2. Extinction spectra for graphene on a photonic crystal in the plasmonic regime in mid-IR range. (a) Measured and (b) simulated extinction spectra of graphene covering a $250 \mathrm{~nm}$ thick photonic crystal membranes with fixed lattice constant of $a=415 \mathrm{~nm}$ and increasing hole radius $(r=80$, $90,100,110,120$, and $140 \mathrm{~nm}$ ). A cumulative vertical offset of $50 \%$ has been included in the spectra for clarity. The peak around $1100 \mathrm{~cm}^{-1}$ comes from the silica material absorption. Inset: Top view of the in-plane electric field profile of the mode corresponding to the peak in a false-color map.
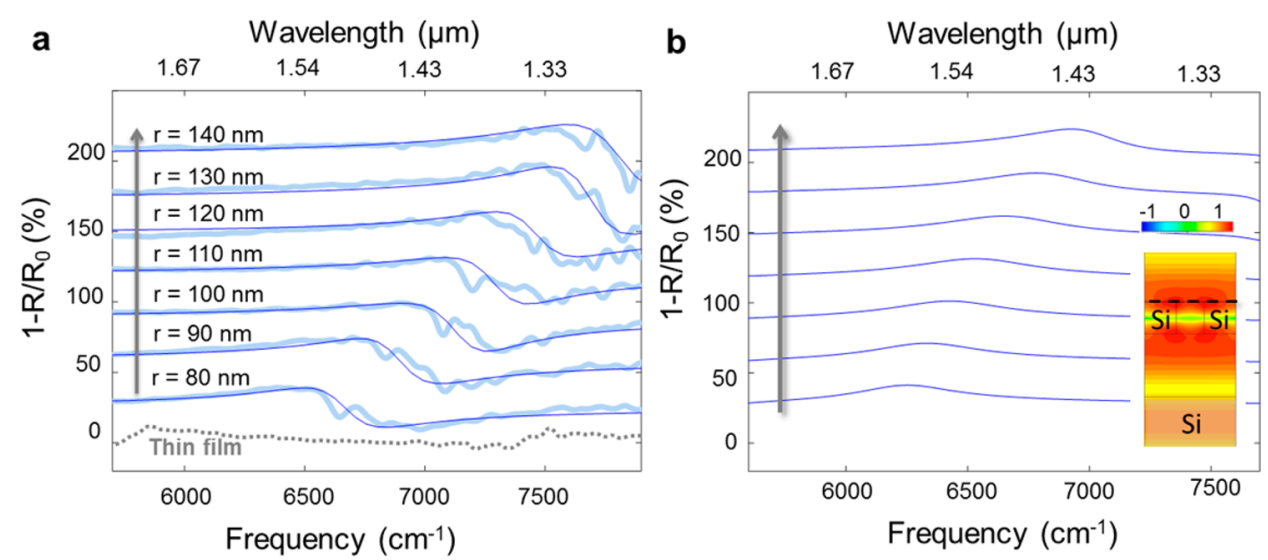

Figure 3. Graphene extinction spectra showing coupling with photonic resonance in the near-IR range. (a) Measured extinction spectra of graphene covering a $250 \mathrm{~nm}$ silicon film (gray dashed curve) and photonic crystal membranes with a fixed lattice constant $a=415 \mathrm{~nm}$ and increasing hole radii from 80 to $140 \mathrm{~nm}$ (light blue). The solid blue curves are based on a Fano resonance model. A vertical offset of $30 \%$ between successive curves has been added for clarity. (b) Numerically simulated extinction spectra for the corresponding photonic crystal membranes. Inset: The vertical electric field amplitude profile for the structure at the resonant frequency. The dashed line shows the position of the graphene layer.

length of the plasmon, the quality factor of the resonator is expected to be relatively low and the resonance broad.

These expectations are confirmed by our experiment and simulations, as shown in Figure 2a,b. The plasmonic peak in the range below $1000 \mathrm{~cm}^{-1}$ exhibits a gradual blue shift with increasing hole radius, that is, with decreasing size of the supported graphene layer. This basic trend, shown by blue arrows in Figure 2, is consistent with excitation of silicongraphene-air plasmonic modes. The inset in Figure $2 \mathrm{~b}$ shows the rapid in-plane spatial oscillations of the electric field distribution at the associated resonance, corresponding to the second-order plasmonic mode in the supported graphene layer. The peak near $1100 \mathrm{~cm}^{-1}$ is associated with a well-known phonon resonance in silica. At this frequency the reflectances $R$ and $R_{0}$ are close to zero, which leads to errors in calculating the extinction ratio $\mathrm{ER}=1-R / R_{0}$. Near $1100 \mathrm{~cm}^{-1}$, a local maximum is predicted in the simulations for a hole radius of 80 $\mathrm{nm}$, while a local minimum is expected for the other hole radii (see Figure $2 \mathrm{~b}$ ). In the experiment spectra, however, only a local maximum is observed (Figure 2a). The strong absorption of silica suppresses the air-graphene-air plasmonic resonances that would otherwise be expected in the range above 1200 $\mathrm{cm}^{-1}$.

\section{PHOTONIC REGIME}

For a Fermi energy of $E_{\mathrm{F}}=0.3 \mathrm{eV}$, interband transitions turn on for frequencies above $4830 \mathrm{~cm}^{-1}$. In the higher-frequency range of 5500-7700 $\mathrm{cm}^{-1}$, where photonic crystal resonances are present, graphene then behaves as an absorptive material with a nearly constant real sheet conductivity of $\sigma_{\mathrm{S}}(\omega)=\sigma_{0}=$ $e^{2} / 4 \hbar$. The measured extinction spectra ER $=1-R / R_{0}$ in Figure 3a exhibit a resonance corresponding to the first FabryPerot photonic resonance (compare the frequency position of the resonance to the kink in effective index of the membrane in Figure S2d). The feature in the extinction spectra exhibits a systematic blue shift with increasing hole radius. The experimental spectra (Figure $3 \mathrm{a}$ ) could be fit well by Fano line shapes.

Figure $3 \mathrm{~b}$ shows the corresponding simulation of our structure. The structure consists of several relatively thick layers (the $250 \mathrm{~nm}$ thick silicon photonic crystal membrane, a $500 \mathrm{~nm}$ thick air gap, and the $1500 \mathrm{~nm}$ thick silica layer above 

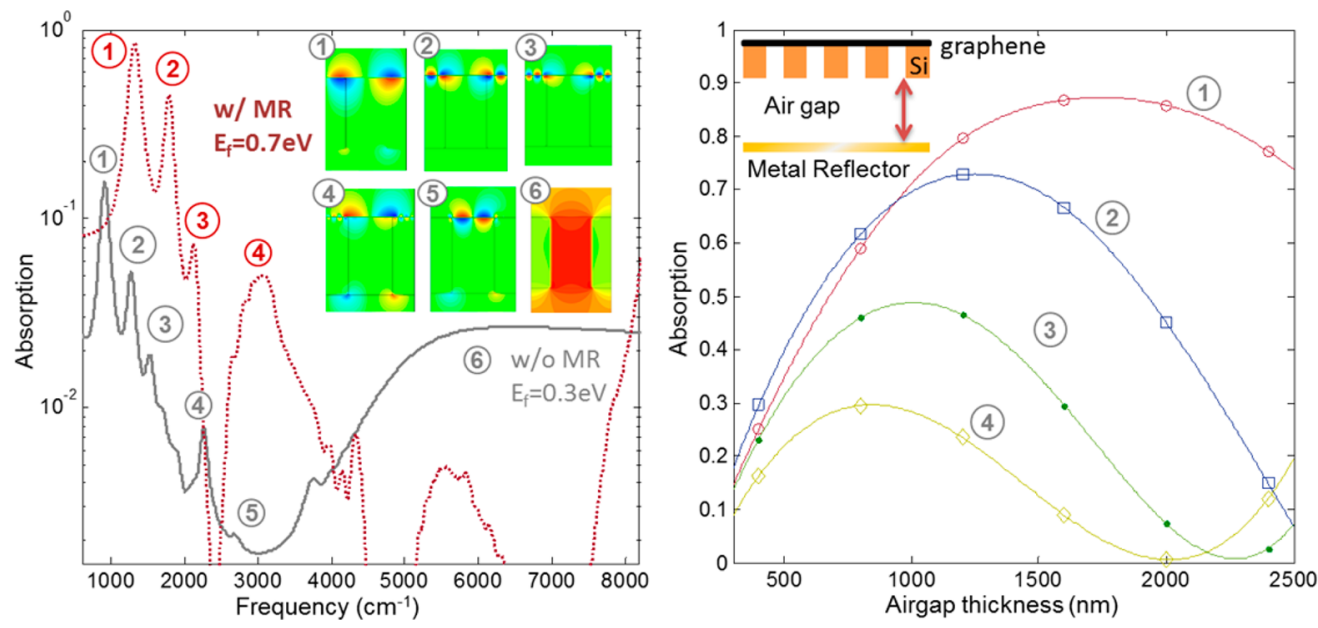

Figure 4. Calculated enhancement of absorption by plasmonic effects in mid-IR range. (a) Absorption of graphene (with $E_{\mathrm{F}}=0.7 \mathrm{eV}$ ) on a silicon photonic crystal membrane $(a=100 \mathrm{~nm}, w / a=0.1, t=100 \mathrm{~nm}$ ) with (red dotted curve) and without (gray solid curve) a back mirror reflector (with an air gap of $1600 \mathrm{~nm}$ ). The field profiles at certain frequencies are shown in the inset. A maximum absorption of $87 \%$ is achieved by increasing Fermi level in graphene to $0.7 \mathrm{eV}$ and setting the air gap thickness to $1600 \mathrm{~nm}$ (see the inset of b). (b) Absorption peak for four plasmonic modes $(1-4$, see panel a) vs the thickness of the air gap.

the bulk silicon substrate, see Figure S1). The presence of these layers leads to multiple resonances in the spectral range of the measurements (see Supporting Information). However, a single resonance has a dominant contribution in the experimental frequency range of $5500-7700 \mathrm{~cm}^{-1}$. The simulations (Figure 3b) are seen to reproduce the observed blue shifts with increasing hole radius. The electric field distribution is illustrated in the inset of Figure $3 \mathrm{~b}$, which shows that the field is concentrated around the graphene layer, thus maximizing its interaction with the photonic mode.

To explore designs for optimized absorbance, we consider graphene on a simplified one-dimensional photonic crystal (Figure 4) with period $a$, thickness $t$, and air hole width $w$. The dependence of the absorbance on these structural parameters is discussed in the Supporting Information S1.3. The electric fields at resonant frequencies represent the whole range of possible surface plasmonic modes (Figure 4a, gray curve), including silicon-graphene-air and air-graphene-air modes, mixed modes, and photonic modes (see the inset for the field maps).

In the case of coupling to the photonic modes, a reasonable approach is to optimize the geometrical parameters so that the photonic modes confined in the photonic crystal plane can achieve higher quality factors. For example, increasing the period $a$ to $500 \mathrm{~nm}$ for a thick silicon membrane of $t=500 \mathrm{~nm}$ causes the absorbance to grow from a few percent to $18 \%$ (see Supporting Information, S1.3). The absorption can be maximized by satisfying the critical coupling criterion in which the photon loss rate from leakage equals the graphene absorption. ${ }^{29,30,47}$ Such solutions are, however, difficult in the plasmonic regime, since plasmonic modes experience greater damping rates.

An alternative approach to increasing the absorbance in both the plasmonic and photonic regimes is to use a back reflector (red curve in Figure 4a). The quality factor of all optical modes (shown in the insets of Figure $4 \mathrm{a}$ ) can be improved using a back reflector, with a controlled air gap between the silicon membrane and metal reflector. For example, adding a metallic mirror with an air spacer of thickness $t_{\text {air }}=1600 \mathrm{~nm}$ below the $t$ $=100 \mathrm{~nm}$ thick membrane with graphene $\left(E_{\mathrm{F}}=0.7 \mathrm{eV}\right)$ induces an increase in absorption from 18 to $87 \%$, as shown in Figure 4a. For higher doping levels, the absorption for each plasmonic mode is maximized for a specific air gap thickness (Figure 4b).

As we have shown in this paper, graphene combined with a photonic crystal membrane offers a rich playground for controlling light-matter interactions in a platform compatible with silicon-based CMOS fabrication technology. We have delineated several different regimes for the enhancement of the absorption of IR radiation of a graphene monolayer placed on a silicon membrane patterned with a two-dimensional photonic crystal lattice. The enhancement, as observed experimentally and predicted theoretically, is a direct consequence of light coupling to plasmonic and photonic modes.

We have classified various regimes of light interaction for the graphene-photonic crystal composite structure: the metamaterial, plasmonic, photonic, and diffraction grating regimes, according to the relation of their effective wavelengths to the structural parameters of the photonic crystal. These different regimes may be suitable for different applications. The metamaterial regime, mainly in the terahertz to mid-IR range, may be of value for tunable absorbers, filters, and modulators. The plasmonic regime in mid-IR can be used, in addition to the aforementioned applications, for sensing due to the extreme confinement of graphene plasmons close to the surface. The photonic regime is suitable for photodetection, since absorption of photons in graphene may generate a photocurrent.

\section{ASSOCIATED CONTENT}

\section{Supporting Information}

The Supporting Information is available free of charge on the ACS Publications website at DOI: 10.1021/acsphotonics.5b00209.

Simulated materials and structures; graphene plasmon properties; absorbance of graphene-covered 1D silicon subwavelength gratings; Raman characterization of the graphene-on-photonic crystal structures; effective index calculations and the reflectivity of multilayer structures; Fano fits to the substrate guided modes in near-IR region (PDF). 


\section{AUTHOR INFORMATION}

\section{Corresponding Author}

*E-mail: tlow@umn.edu.

\section{Present Address}

$\square$ Electrical Engineering Department, University of Minnesota, MN 55455.

\section{Author Contributions}

${ }^{\dagger}$ These authors contributed equally (T.G. and A.A.).

\section{Notes}

The authors declare no competing financial interest.

\section{ACKNOWLEDGMENTS}

The authors thank Z. Huang and C. Li at Hewlett-Packard Laboratories for valuable discussions and Carlos Forsythe at Columbia University for assistance with the AFM measurements. Funding from the National Science Foundation is gratefully acknowledged (Grant CBET-1438147 for support of C.W.W. and Grant DMR-1411107 for support of T.L. and Y.L.). A.A. acknowledges financial support from the Danish Council for Technical and Production Sciences through the GraTer Project (Contract No. 0602-02135B).

\section{REFERENCES}

(1) Reed, G. T.; Knights, A. P. Silicon Photonics; Wiley Online Library: New York, 2008.

(2) Krier, A. Mid-Infrared Semiconductor Optoelectronics; Springer: New York, 2006.

(3) Bonaccorso, F.; Sun, Z.; Hasan, T.; Ferrari, A. C. Graphene photonics and optoelectronics. Nat. Photonics 2010, 4, 611-622.

(4) Soref, R. Mid-infrared photonics in silicon and germanium. Nat. Photonics 2010, 4, 495-497.

(5) Jablan, M.; Buljan, H.; Soljačić, M. Plasmonics in graphene at infrared frequencies. Phys. Rev. B: Condens. Matter Mater. Phys. 2009, $80,245435$.

(6) Grigorenko, A.; Polini, M.; Novoselov, K. Electrically controlled one-way photon flow in plasmonic nanostructures. Nat. Photonics 2012, 6, 749-758.

(7) Yan, H.; Li, X.; Chandra, B.; Tulevski, G.; Wu, Y.; Freitag, M.; Zhu, W.; Avouris, P.; Xia, F. Tunable infrared plasmonic devices using graphene/insulator stacks. Nat. Nanotechnol. 2012, 7, 330-334.

(8) Fei, Z.; Rodin, a S.; Andreev, G. O.; Bao, W.; McLeod, a S.; Wagner, M.; Zhang, L. M.; Zhao, Z.; Thiemens, M.; Dominguez, G.; Fogler, M. M.; Castro Neto, a H.; Lau, C. N.; Keilmann, F.; Basov, D. $\mathrm{N}$. Gate-tuning of graphene plasmons revealed by infrared nanoimaging. Nature 2012, 487, 82-85.

(9) Chen, J.; Badioli, M.; Alonzo-Gonzalez, P.; Thongrattanasiri, S.; Huth, F.; Osmond, J.; Spasenović, M.; Centeno, A.; Pesquera, A.; Godignon, P.; Zurutuza Elorza, A.; Camara, N.; Hillenbrand, R.; Koppens, F. H. L.; Alonso-González, P.; Elorza, A. Z.; García de Abajo, F. J. Optical nano-imaging of gate-tunable graphene plasmons. Nature 2012, 487, 77-81.

(10) Alonso-González, P.; Nikitin, a Y.; Golmar, F.; Centeno, a; Pesquera, a; Vélez, S.; Chen, J.; Navickaite, G.; Koppens, F.; Zurutuza, a; Casanova, F.; Hueso, L. E.; Hillenbrand, R. Controlling graphene plasmons with resonant metal antennas and spatial conductivity patterns. Science 2014, 344, 1369-1373.

(11) Yan, H.; Low, T.; Zhu, W.; Wu, Y.; Freitag, M.; Li, X.; Guinea, F.; Avouris, P.; Xia, F. Damping pathways of mid-infrared plasmons in graphene nanostructures. Nat. Photonics 2013, 7, 394-399.

(12) Ju, L.; Geng, B.; Horng, J.; Girit, C.; Martin, M.; Hao, Z.; Bechtel, H. A.; Liang, X.; Zettl, A.; Shen, Y. R.; Wang, F. Graphene plasmonics for tunable terahertz metamaterials. Nat. Nanotechnol. 2011, 6, 630-634.

(13) Low, T.; Avouris, P. Graphene Plasmonics for Terahertz to MidInfrared Applications. ACS Nano 2014, 8, 1086-1101.
(14) Bao, Q.; Loh, K. P. Graphene Photonics, Plasmonics, and Broadband Optoelectronic Devices. ACS Nano 2012, 6, 3677-3694.

(15) Sensale-Rodriguez, B.; Yan, R.; Kelly, M. M. M.; Fang, T.; Tahy, K.; Hwang, W. S.; Jena, D.; Liu, L.; Xing, H. G. Enhancing and inhibiting stimulated Brillouin scattering in photonic integrated circuits. Nat. Commun. 2012, 3, 780-787.

(16) Li, W.; Chen, B.; Meng, C.; Fang, W.; Xiao, Y.; Li, X.; Hu, Z.; Xu, Y.; Tong, L.; Wang, H.; Liu, W.; Bao, J.; Shen, Y. R. Ultrafast alloptical graphene modulator. Nano Lett. 2014, 14, 955-959.

(17) Liu, M.; Yin, X.; Ulin-Avila, E.; Geng, B.; Zentgraf, T.; Ju, L.; Wang, F.; Zhang, X. A graphene-based broadband optical modulator. Nature 2011, 474, 64-67.

(18) Li, Y.; Yan, H.; Farmer, D. B.; Meng, X.; Zhu, W.; Osgood, R. M.; Heinz, T. F.; Avouris, P. Graphene Plasmon Enhanced Vibrational Sensing of Surface-Adsorbed Layers. Nano Lett. 2014, 14, 1573-1577.

(19) Youngblood, N.; Anugrah, Y.; Ma, R.; Koester, S. J.; Li, M. Multifunctional Graphene Optical Modulator and Photodetector Integrated on Silicon Waveguides. Nano Lett. 2014, 14, 2741-2746.

(20) Nene, P.; Strait, J. H.; Chan, W.-M.; Manolatou, C.; Tiwari, S.; McEuen, P. L.; Rana, F. Coupling of plasmon modes in graphene microstructures. Appl. Phys. Lett. 2014, 105, 143108.

(21) Brar, V.; Jang, M.; Sherrott, M. Highly Confined Tunable MidInfrared Plasmonics in Graphene Nanoresonators. Nano Lett. 2013, $13,2541-2547$.

(22) Nikitin, A. Y.; Guinea, F.; Martin-Moreno, L. Resonant plasmonic effects in periodic graphene antidot arrays. Appl. Phys. Lett. 2012, 101, 151119.

(23) Zhu, X.; Wang, W.; Yan, W.; Larsen, M. Plasmon-phonon coupling in large-area graphene dot and antidot arrays fabricated by nanosphere lithography. Nano Lett. 2014, 14, 2907-2913.

(24) Yeung, K. Y. M.; Chee, J.; Yoon, H.; Song, Y.; Kong, J.; Ham, D. Far-infrared graphene plasmonic crystals for plasmonic band engineering. Nano Lett. 2014, 14, 2479-2484.

(25) Wang, M.; Fu, L.; Gan, L.; Zhang, C.; Rümmeli, M.; Bachmatiuk, A.; Huang, K.; Fang, Y.; Liu, Z. CVD growth of large area smooth-edged graphene nanomesh by nanosphere lithography. Sci. Rep. 2013, 3, 1238.

(26) Gao, W.; Shu, J.; Qiu, C.; Xu, Q. Excitation of Plasmonic Waves in Graphene by Guided-Mode Resonances. ACS Nano 2012, 6, 78067813.

(27) Zhu, X.; Yan, W.; Uhd Jepsen, P.; Hansen, O.; Asger Mortensen, N.; Xiao, S. Experimental observation of plasmons in a graphene monolayer resting on a two-dimensional subwavelength silicon grating. Appl. Phys. Lett. 2013, 102, 131101

(28) Low, T.; Perebeinos, V.; Kim, R.; Freitag, M.; Avouris, P. Cooling of photoexcited carriers in graphene by internal and substrate phonons. Phys. Rev. B: Condens. Matter Mater. Phys. 2012, 86, 045413.

(29) Piper, J. R.; Fan, S. Total Absorption in a Graphene Monolayer in the Optical Regime by Critical Coupling with a Photonic Crystal Guided Resonance. ACS Photonics 2014, 1, 347-353.

(30) Liu, Y.; Chadha, A.; Zhao, D.; Piper, J. R.; Jia, Y.; Shuai, Y.; Menon, L.; Yang, H.; Ma, Z.; Fan, S.; Xia, F.; Zhou, W. Approaching total absorption at near infrared in a large area monolayer graphene by critical coupling. Appl. Phys. Lett. 2014, 105, 181105.

(31) Gan, X.; Mak, K.; Gao, Y.; You, Y.; Hatami, F. Strong Enhancement of Light-Matter Interaction in Graphene Coupled to a Photonic Crystal Nanocavity. Nano Lett. 2012, 12, 5626-5631.

(32) Gan, X.; Mak, K.; Gao, Y.; You, Y.; Hatami, F. High-Contrast Electrooptic Modulation of a Photonic Crystal Nanocavity by Electrical Gating of Graphene. Nano Lett. 2013, 13, 691-696.

(33) Majumdar, A.; Kim, J.; Vuckovic, J.; Wang, F. Electrical control of silicon photonic crystal cavity by graphene. Nano Lett. 2013, 13, 515-518.

(34) Kong, X.-T.; Bai, B.; Dai, Q. Graphene plasmon propagation on corrugated silicon substrates. Opt. Lett. 2015, 40, 1-4.

(35) Andryieuski, A.; Lavrinenko, A. V. Graphene metamaterials based tunable terahertz absorber: effective surface conductivity approach. Opt. Express 2013, 21, 9144. 
(36) Chatterjee, R.; Panoiu, N.; Liu, K.; Dios, Z.; Yu, M.; Doan, M.; Kaufman, L.; Osgood, R.; Wong, C. Achieving Subdiffraction Imaging through Bound Surface States in Negative Refraction Photonic Crystals in the Near-Infrared Range. Phys. Rev. Lett. 2008, 100, 187401.

(37) Hao, Y.; Bharathi, M. S.; Wang, L.; Liu, Y.; Chen, H. S.; Nie, X.; Wang; Chou, H.; Tan, C.; Fallahazad, B.; Ramanarayan, H.; Magnuson, C. W.; Tutuc, E.; Yakobson, B. I.; McCarty, K. F.; Zhang, Y.-W.; Kim, P.; Hone, J.; Colombo, L.; Ruoff, R. S. The Role of Surface Oxygen in the Growth of Large Single-Crystal Graphene on Copper. Science 2013, 342, 720-723.

(38) Hao, Y.; Wang, Y.; Wang, L.; Ni, Z.; Wang, Z.; Wang, R.; Koo, C. K.; Shen, Z.; Thong, J. T. L. Probing Layer Number and Stacking Order of Few-Layer Graphene by Raman Spectroscopy. Small 2010, 6, 195-200.

(39) Berciaud, S.; Ryu, S.; Brus, L. E.; Heinz, T. F. Probing the intrinsic properties of exfoliated graphene: Raman spectroscopy of free-standing monolayers. Nano Lett. 2009, 9, 346-352.

(40) CST, Computer Simulation Technology, AS; CST of America: Framingham, MA; http://cst.com.

(41) Palik, E. D.; Ghosh, G. Handbook of Optical Constants of Solids; Academic Press: Waltham, MA, 1998.

(42) Kitamura, R.; Pilon, L.; Jonasz, M. Optical constants of silica glass from extreme ultraviolet to far infrared at near room temperature. Appl. Opt. 2007, 46, 8118.

(43) Mason, D. R.; Menabde, S. G.; Park, N. Unusual Otto excitation dynamics and enhanced coupling of light to TE plasmons in graphene. Opt. Express 2014, 22, 847.

(44) Gao, W.; Shu, J.; Reichel, K.; Nickel, D. V.; He, X.; Shi, G.; Vajtai, R.; Ajayan, P. M.; Kono, J.; Mittleman, D. M.; Xu, Q. HighContrast Terahertz Wave Modulation by Gated Graphene Enhanced by Extraordinary Transmission through Ring Apertures. Nano Lett. 2014, 14, 1242-1248.

(45) Hafez, H. a.; Al-Naib, I.; Oguri, K.; Sekine, Y.; Dignam, M. M.; Ibrahim, a.; Cooke, D. G.; Tanaka, S.; Komori, F.; Hibino, H.; Ozaki, T. Nonlinear transmission of an intense terahertz field through monolayer graphene. AIP Adv. 2014, 4, 117118.

(46) Buron, J.; Pizzocchero, F.; Jessen, B.; Booth, T. J.; Nielsen, P. F.; Hansen, O.; Hilke, M.; Whiteway, E.; Jepsen, P. U.; Bøggild, P.; Petersen, D. H. Electrically Continuous Graphene from Single Crystal Copper Verified by Terahertz Conductance Spectroscopy and Micro Four-Point Probe. Nano Lett. 2014, 14, 6348-6355.

(47) Huang, L., Yu, Y., Cao L., Ultrathin semiconductor perfect light absorbers with high spectral, polarization, and angle selectivity for arbitrary wavelengths. arXiv:1404.6842, 2014. 